\title{
Raman Polarizer Based on a Fiber with a Random Birefringence
}

\author{
Auro M. Perego ${ }^{1, *}$ and Sergey V. Sergeyev ${ }^{1}$ \\ 1. Aston Institute of Photonic Technologies, Aston University, Birmingham, B4 7ET, UK \\ "peregoa@aston.ac.uk
}

Raman polarizers are devices able to amplify and simultaneously repolarize optical signals, exploiting the polarization attraction phenomenon induced by the Raman gain anisotropy. Up to now a proper analytical description the signal degree of polarization (DOP) for Raman polarizers has not been formulated yet. Only some approximated expressions have been derived, which however have limited validity [1,2], in particular they don't include the dependence on the initial signal and pump relative state of polarization (SOP) orientation, nor on the polarization mode dispersion (PMD) parameter $D_{p}$. Starting from the rigorously derived averaged polarizer equations [3-5], we have obtained a formula for the forward pumped Raman polarizer DOP, easily generalizable to different pumping schemes and valid in the limit of negligible pump depletion and self/cross-phase modulation:

$$
D O P=\sqrt{1-\exp \left(-g P_{i n} \int_{0}^{z}\langle x\rangle d z^{\prime}\right)}
$$

In Eq. 1, $g$ represents the Raman gain, $P_{i n}$ the input pump power, $z$ is the spatial coordinate and $\langle x\rangle=\langle\vec{S} \cdot \hat{p}\rangle /\left\langle s_{0}\right\rangle$; being $\vec{s}$ the signal Stokes vector, $\hat{p}$ the unit vector describing the pump state of polarization, $s_{0}$ the signal intensity while bra-kets denote averaged quantities. Our formula includes properly the initial relative pump and signal SOPs orientation, as well as the impact of the PMD parameter and birefringence correlation length from which $\langle x\rangle$ implicitly depends.

In Fig. 1 we have represented the polarizer DOP as a function of fiber length, signal gain and birefringence correlation length, for various values of the PMD parameter. We have computed $\langle x\rangle$ numerically using the coupled averaged equations for the Raman polarizer [3-5].
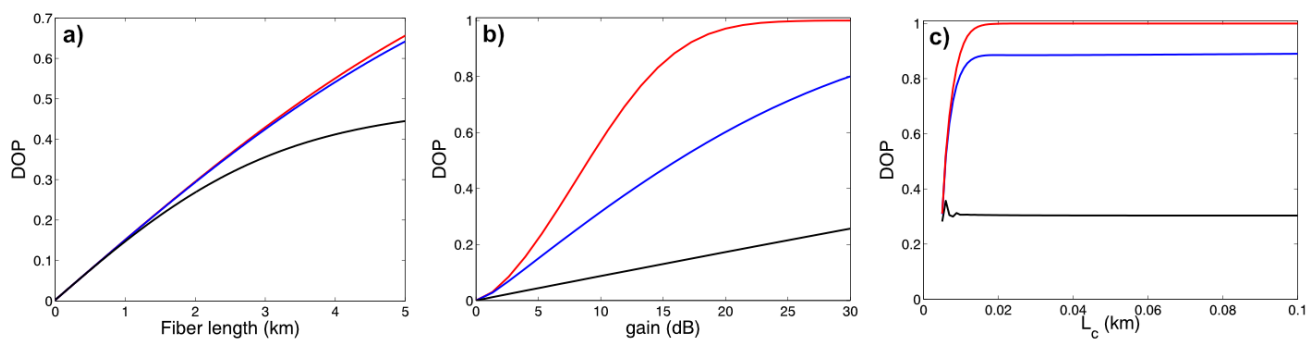

Fig. 1 Signal DOP is plotted versus fiber length $L$ a), amount of gain b) and birefringence correlation length $L_{c}$ c); for different values of the PMD parameter (red, blue and black lines correspond to $D_{p}=0.005,0.05$ and $0.2 \mathrm{ps} \bullet \mathrm{km}^{-1 / 2}$ respectively). In a) we have chosen $L_{c}=0.01 \mathrm{~km}$ and $P_{i n}=5 \mathrm{~W}$; in b) $L_{c}=0.01 \mathrm{~km}$ and $P_{\text {in }}$ has been varied from 0 to $2.2 \mathrm{~W}$; in c) $L=3 \mathrm{~km}$ and $P_{\text {in }}=5 \mathrm{~W}$. In all the plots we have taken $g=0.6(\mathrm{~km} \bullet \mathrm{W})^{-1}$, pump and signal wavelengths 1450 and $1550 \mathrm{~nm}$ respectively, input signal power $1 \mathrm{~mW}$; furthermore we have assumed negligible signal losses and uncorrelated pump and signal SOP at the beginning of the fields propagation in the polarizer.

Our preliminary study suggests the possibility of an interesting systematic investigation of the DOP dependence on the PMD parameter and on the fiber correlation length. The new formula is general and can be easily generalized also to different pumping schemes (backward and simultaneous forward-backward pumping) and compared realistically with experimental results. Analytic asymptotic solutions can be obtained too and results will be communicated soon.

\section{References}

[1] V. V. Kozlov, J. Nuño, J. D. Ania-Castañon and S. Wabnitz, "Trapping polarization of light in nonlinear optical fibers: an ideal Raman polarizer", Chapter in Spontaneous Symmetry Breaking, Self-Trapping, and Josephson Oscillations, B. A. Malomed ed. (Springer, Berlin, 2012).

[2] F. Chiarello, L. Palmieri, M. Santagiustina, R. Gamatham and A. Galtarossa, "Experimental characterization of the counter-propagating Raman polarization attraction", Optics Express 20, 26050 (2012).

[3] V. Kalashnikov, S. V. Sergeyev, G. Jacobsen, S. Popov and S. K. Turitsyn, "Multi-scale polarisation phenomena”, Light: Science \& Applications 5, e16011 (2016).

[4] V. Kalashnikov, S. V. Sergeyev, G. Jacobsen, J. D. Ania-Castañon and S. Popov, "Stochastic phenomena in a fiber Raman amplifier", Annalen der Physik, 1-11 (DOI 10.1002) (2016).

[5] S. V. Sergeyev, S. Popov and A. T. Friberg, "Modelling polarization-dependent gain in fiber Raman amplifiers with randomly varying birefringence", Optics Communications 262, 114 (2006). 